\title{
Geschäftsanalyse im Kontext der Unternehmensarchitektur
}

Die Wandlungsfähigkeit von Organisationen ist ein wesentlicher Erfolgsfaktor, zu dem die Unternehmensarchitektur einen wichtigen Beitrag leisten kann. Geschäftsanalysen liefern die Voraussetzungen für das Verständnis und die transparente Weiterentwicklung des Geschäftsmodells und die dafür notwendige Ausrichtung der Informationstechnologie. Vor diesem Hintergrund wird am Beispiel eines Schweizerischen Versicherungsunternehmens gezeigt, wie durch Geschäftsanalyse einerseits das Verständnis des Geschäftsmodells gefördert und andererseits die Abstimmung zwischen Geschäfts- und IT-Architektur unterstützt wird. Ferner wird ein Ansatz vorgestellt, der verschiedene Ziele und Typen der Geschäftsanalyse im Kontext von Unternehmensarchitekturen zusammenführt.

\section{Inhaltsübersicht}

1 Grundlagen der Geschäftsanalyse

1.1 Unternehmensarchitektur, Architekturebenen und Gestaltungsobjekte

1.2 Geschäftsarchitektur als Basis der Geschäftsanalyse

2 Ziele und Einsatzszenarien der Geschäftsanalyse

2.1 Zielorientierte Geschäftsanalyse

2.2 Einsatzszenarien der Geschäftsanalyse

3 Fallbeispiel AXA Winterthur

3.1 Allgemeine Ziele der

Geschäftsarchitektur

3.2 Geschäftsanalyse aus Sicht der strategischen Unternehmensführung

3.3 Geschäftsanalyse aus Sicht der Produktentwicklung

3.4 Werkzeugunterstützte Geschäftsanalyse
4 Gesamtmodell der Geschäftsanalyse 4.1 Typen der Geschäftsanalyse 4.2 Breite und Tiefe der Geschäftsanalyse

4.3 Umsetzung der Geschäftsanalyse

5 Lerneffekte und weitere Entwicklung

6 Literatur

\section{Grundlagen der Geschäftsanalyse}

Um in einem sich kontinuierlich verändernden Marktumfeld langfristig bestehen zu können, müssen Unternehmen eine hohe Flexibilität, Reaktionsgeschwindigkeit und Innovationsfähigkeit aufweisen. Dabei nehmen das Ausmaß und das Tempo der dafür notwendigen Transformation im Unternehmen zu. Dies führt in erster Linie zu steigender Komplexität und Dynamik der Unternehmensarchitektur sowie zu einer wachsenden Anzahl von Abhängigkeiten. Eine zentrale Herausforderung besteht darin, Transparenz, bezogen auf das Unternehmen als Ganzes, zu gewinnen, d. h. die Zusammenhänge, die für die Abwicklung des Geschäfts zentral sind, verstehen und zielgerichtet analysieren zu können. Im Zeitverlauf sind oft heterogene, sehr komplexe Architekturen entstanden, deren Steuerung und Weiterentwicklung im Sinne der adäquaten Unterstützung des Geschäfts oftmals mit hohem Aufwand verbunden sind. Ein erster wichtiger Schritt zur Schaffung von Transparenz und zur zweckmäßigen Ausrichtung der IT auf fachliche Anforderungen besteht darin, die Geschäftsanalyse im Kontext der Unternehmensarchitektur zu forcieren. Mithilfe von Geschäftsanalysen wird das Geschäftsmodell transparent gemacht. Änderungen können frühzeitig identifiziert und deren Auswirkungen können strukturiert beurteilt werden. Damit wird die Grundlage für systema- 
tisches Business-IT-Alignment und somit für die Flexibilisierung des Unternehmens geschaffen.

\subsection{Unternehmensarchitektur, Architektur- ebenen und Gestaltungsobjekte}

Die Unternehmensarchitektur stellt ein aggregiertes Gesamtbild einer Organisation dar mit dem Ziel, die Gesamtzusammenhänge zu strukturieren und gleichzeitig den Korridor für die zukünftige Entwicklung und Gestaltung aufzuzeigen. Sie wird nicht durch ein einziges Architekturmodell, sondern durch eine Menge von Teilarchitekturen beschrieben. Je nach Verwendungszweck und Zielgruppe werden dabei Gestaltungsobjekte bestimmten Architekturebenen zugeordnet [Baumöl 2006; Lankhorst 2005; The Open Group 2007]. In [Winter \& Fischer 2007] werden verschiedene Unternehmensarchitekturansätze im Hinblick auf die jeweils vorgeschlagenen Architekturebenen analysiert. Die dort betrachteten Ansätze lassen sich in Form der in Abbildung 1 illustrierten Architekturebenen konsolidieren. In Abbildung 1 werden jeweils auch exemplarische Gestaltungsobjekte der jeweiligen Unternehmensarchitekturebene aufgeführt.

Die Teilarchitekturen der Unternehmensarchitektur haben unterschiedliche Lebenszyklen. Während die grundlegenden IT-Strukturen
(Software- und Infrastrukturebene) im Normalfall durch relative Stabilität gekennzeichnet sind, sind die fachlichen Strukturen (Strategieund Organisationsebene) vergleichsweise häufigeren und oft auch massiven Änderungen unterworfen. Die Unterscheidung und Entkopplung von Teilarchitekturen erlaubt, diese unterschiedliche Dynamik in der Unternehmensarchitektur abzubilden.

\subsection{Geschäftsarchitektur als Basis der Geschäftsanalyse}

Ein im Kontext der Unternehmensarchitektur wenig adressierter Bereich ist die Geschäftsarchitektur [Winter et al. 2007]. Als Teilarchitektur der Unternehmensarchitektur bildet sie die fachlichen Gestaltungsobjekte eines Unternehmens implementierungsneutral $a b$. Gemäß dem Unternehmensarchitekturverständnis aus Abbildung 1 umfasst die Geschäftsarchitektur Gestaltungsobjekte auf Strategie-, Organisations- und teilweise auch auf Integrationsebene. Sie beschreibt die fachlichen Grundstrukturen bzgl. der Leistungen, Geschäftsziele, Geschäftsprozesse und Organisationsstrukturen sowie der dafür notwendigen Ressourcen [Keller 2007]. Die Geschäftsarchitektur bildet somit die Basis der Geschäftsanalyse. Geschäftsanalysen im Kontext der Unternehmensarchitektur

\begin{tabular}{|c|c|c|}
\hline $\begin{array}{l}\text { Strategie- } \\
\text { ebene }\end{array}$ & $\begin{array}{l}\text { - } \quad \text { Geschäftsnetzwerk } \\
\text { - } \quad \text { Ziele und Erfolgsfaktoren } \\
\text { - } \quad \text { Produkt-/Leistungsmodell }\end{array}$ & $\begin{array}{l}\text { - } \quad \text { Märkte bzw. Marktsegmente } \\
\text { - } \quad \text { Kernkompetenzen } \\
\text { - } \quad \text { Strategische Programme/Projekte }\end{array}$ \\
\hline $\begin{array}{l}\text { Organisations- } \\
\text { ebene }\end{array}$ & $\begin{array}{l}\text { - Geschäftsfunktionen } \\
\text { - Geschäftsprozesse } \\
\text { - Kennzahlen }\end{array}$ & $\begin{array}{l}\text { - } \quad \text { Leistungsflüsse } \\
\text { - Organisationseinheiten \& Rollen } \\
\text { - } \quad \text { Informationsobjekte \& -flüsse }\end{array}$ \\
\hline $\begin{array}{l}\text { Integrations- } \\
\text { ebene }\end{array}$ & $\begin{array}{l}\text { - Domänen } \\
\text { - Applikationen und Applikationskomponenten }\end{array}$ & $\begin{array}{l}\text { - Spezifikation der Enterprise Services und } \\
\text { Servicekomponenten }\end{array}$ \\
\hline $\begin{array}{l}\text { Software- } \\
\text { ebene }\end{array}$ & $\begin{array}{l}\text { - Softwarekomponenten: } \\
\text { Funktionshierarchie etc. }\end{array}$ & $\begin{array}{l}\text { - Spezifikation der Datenressourcen: } \\
\text { Logische und physische Datenmodelle }\end{array}$ \\
\hline $\begin{array}{l}\text { Infrastruktur- } \\
\text { ebene }\end{array}$ & $\begin{array}{l}\text { Spezifikation der IT-Komponenten: Plattforme } \\
\text { Hardware- und Netzwerkkomponenten etc. }\end{array}$ & \\
\hline
\end{tabular}

Abb. 1: Ebenen der Unternehmensarchitektur 
stellen einen hohen Nutzen in Aussicht, da die einbezogenen Gestaltungsobjekte oftmals redundant abgebildet werden (z. B. in Produktmanagementsystemen, Performance-Management-Systemen, Prozessmanagementsystemen, HR-Systemen) und die Verknüpfungen der relevanten Gestaltungsobjekte häufig unklar sind. Ausgehend vom Geschäftsmodell, das im Zusammenhang mit der Gesamtstrategie des betreffenden Unternehmens zu betrachten ist, können so zielgerichtet Veränderungen lokalisiert werden. Implikationen für die Weiterentwicklung bewegen sich dabei u.a. im Rahmen der Konsolidierung und Standardisierung von Geschäftsprozessen. Des Weiteren bietet die Geschäftsanalyse Einblick in mögliche Auswirkungen der Geschäftsentwicklung und ist somit die geeignete Grundlage für eine koordinierte Weiterentwicklung der fachlichen und IT-Architekturen. Die Umsetzung der Unternehmensarchitektur als passives Repository der im Unternehmen vorhandenen Artefakte greift aus den genannten Gründen zu kurz. Vielmehr muss die Analyse von Geschäftsarchitekturen zur Optimierung bestehender Strukturen und Abläufe aktiv forciert werden. Damit kommt der Unternehmensarchitektur eine wichtige moderierende, teilweise auch gestaltende Funktion zu.

\section{Ziele und Einsatzszenarien der Geschäftsanalyse}

Die Verwendung von Geschäftsanalysen im Kontext der Unternehmensarchitektur sollte zielorientiert erfolgen.

\subsection{Zielorientierte Geschäftsanalyse}

Analysen werden allgemein zum Zweck der Überwachung und Optimierung verwendet. Damit eine effektive Steuerung in Richtung des gewünschten Zustands möglich ist, müssen zunächst erreichbare Ziele definiert werden. Im Kontext der Unternehmensarchitektur können anhand der Konsolidierung bestehender Archi- tekturziele aus Literatur und Praxis z. B. die folgenden fünf aufeinander aufbauenden Stufen identifiziert werden:

Stufe 1: Transparenz. Das Analyseziel besteht darin, die Unternehmensarchitektur zu verstehen und nachzuvollziehen. Dies geschieht hauptsächlich, indem veraltete bzw. inkonsistente Dokumentationen strukturiert sowie bzgl. der Istsituation im Unternehmen aktualisiert werden. Dadurch wird die Grundlage geschaffen, um die Weiterentwicklung von Gestaltungsobjekten nachvollziehbar und konform mit den tatsächlichen Strukturen zu steuern.

Stufe 2: Komplexitätsbeherrschung. Die Geschäftsanalyse strebt an, Strukturen zu vereinfachen und zu konsolidieren. Architekturen homogener zu gestalten ist u.a. möglich, indem auf relevante Standards zurückgegriffen wird. Eine verminderte Komplexität erlaubt es, Systeme skalierbar und mit erhöhter Stabilität zu betreiben. Eine handhabbare Anzahl von Schnittstellen in der Unternehmensarchitektur gewährleistet zudem, dass zukünftige Änderungen von (Teil-)Architekturen mit vertretbarem Aufwand umgesetzt werden können.

Stufe 3: Konsistenzerhaltung. Auf der dritten Stufe besteht das Analyseziel darin, die Kohärenz der Unternehmensarchitektur zu erhöhen. Dazu wird der optimale Grad der fachlichen Integration und des Alignments von Gestaltungsobjekten untersucht. Entsprechende Analysen liefern hier wichtigen Input, u. a. um mögliche Redundanzen zu minimieren.

Stufe 4: Wandlungsfähigkeit. Auf der vierten Stufe steht das Ziel der Wandlungsfähigkeit im Mittelpunkt. Geschäftsanalysen können zeigen, wo Potenziale für systematische Wiederverwendung liegen und durch welche Strukturierungen die Flexibilität erhöht werden kann. Die Reaktionsfähigkeit des Unternehmens wird darüber hinaus beeinflusst, inwiefern nicht antizipierte Änderungen umsetzbar sind. Hierbei wird das Analyseziel der Agilität hervorgehoben. 
Stufe 5: Nachhaltigkeit. Die Analyse im Sinne einer nachhaltigen Unternehmensentwicklung legt den Fokus auf die langfristigen Auswirkungen von Änderungen. Es wird eine Balance angestrebt zwischen der Wirtschaftlichkeit und der Aufrechterhaltung der Innovationsfähigkeit im Unternehmen, um im sich kontinuierlich verändernden Marktumfeld dauerhaft bestehen zu können.

Mithilfe der dargestellten Ziele kann eine Zielhierarchie konstruiert werden. Hierbei bedingt das Erreichen einer höheren Hierarchiestufe die Erfüllung von Anforderungen der Vorstufe (vgl. Abb. 2).

\subsection{Einsatzszenarien der Geschäftsanalyse}

Für Unternehmensarchitekturen haben sich verschiedenste Einsatzszenarien etabliert [Keller 2007; Niemann 2005; Winter et al. 2007]. Die Abgrenzung der einzelnen Szenarien ist dabei nicht immer überschneidungsfrei. Tabelle 1 zeigt exemplarisch die Aufgabengebiete, in deren Rahmen sich die Nutzung der Geschäftsanalyse insbesondere empfiehlt.

So fokussiert z. B. ein Business-ContinuityProjekt auf die Gewährleistung der Verfügbarkeit von Applikationen und Produkten, die durch das Architekturziel "Sicherstellung von Transparenz« auf Stufe 1 unterstützt wird. Ent- scheidungsprozesse, wie sie in den genannten Einsatzszenarien existieren, können mit Ergebnissen der Geschäftsanalyse angereichert werden. Die Analyse fachlicher Zusammenhänge unterstützt die Adressaten in ihrem jeweiligen Aufgabengebiet, Ergebnisse transparent zu gewinnen. Damit wird die konsistente und nachhaltige Weiterentwicklung der involvierten Gestaltungsobjekte unterstützt.

\section{Fallbeispiel AXA Winterthur}

Nachfolgend wird die Geschäftsanalyse auf Basis der Unternehmensarchitektur am Beispiel einer Initiative zur Erstellung und Pflege der Geschäftsarchitektur der AXA Winterthur vorgestellt.

\subsection{Allgemeine Ziele der Geschäfts- architektur}

Die AXA Winterthur ist seit dem 1. Januar 2007 die Schweizer Tochtergesellschaft der AXA. Mit 52 Millionen Kunden und einem Umsatz von 79 Mrd. Euro ist die AXA einer der weltweit führenden Versicherungskonzerne. Innerhalb dieser Gruppe repräsentiert die AXA Winterthur die Ländereinheit Schweiz. Die Integration der Winterthur-Versicherungen in den neuen Rahmenkonzern bedeutete auch die Integration in dessen strategische Geschäftsführung. Eine

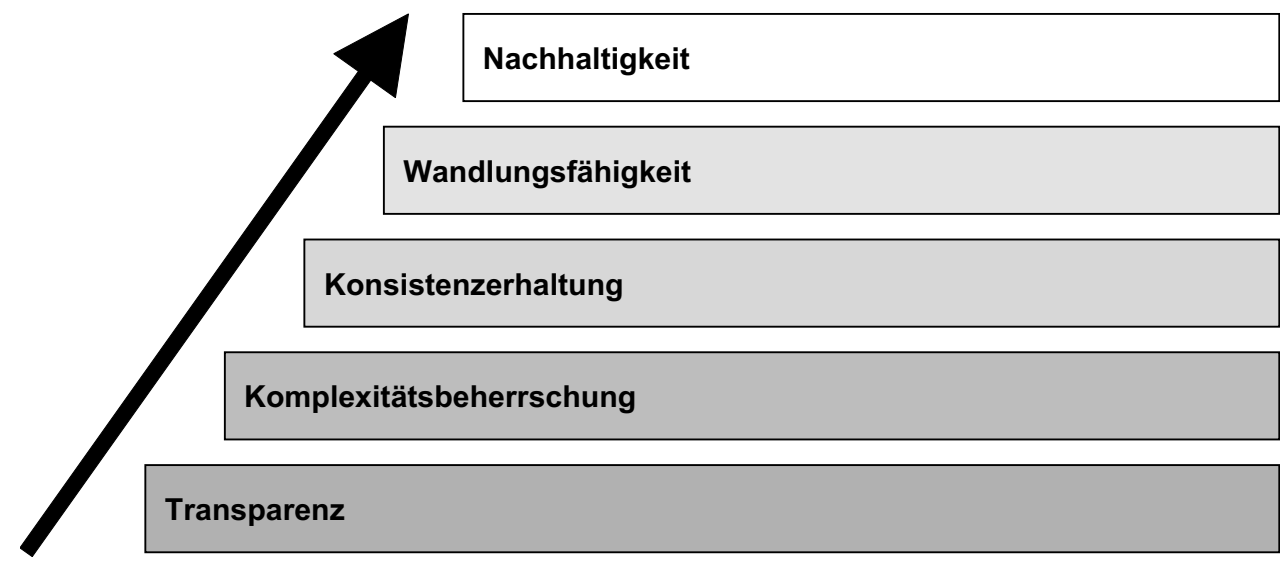

Abb. 2: Zielhierarchie für Geschäftsanalysen 


\begin{tabular}{|l|l|l|l|}
\hline Einsatzszenario & Adressat & Gestaltungsobjekte & Ziele \\
\hline Business-IT-Alignment & Geschäftsleitung & Domänen, fachliche Services & Stufe 3 \\
\hline Business Continuity Planning & Operative Leitung & Applikationen, Produkte & Stufe 1 \\
\hline Projektportfolioplanung & Projektmanagement & $\begin{array}{l}\text { Strategische Programme und } \\
\text { Projekte, Unternehmensziele }\end{array}$ & Stufe 5 \\
\hline Produktplanung & Produktmanager & $\begin{array}{l}\text { Produkte, Markt- und } \\
\text { Kundensegmente }\end{array}$ & Stufe 4 \\
\hline Prozessoptimierung & Fachbereiche & $\begin{array}{l}\text { Geschäftsprozesse, } \\
\text { Geschäftsfunktionen, Rollen }\end{array}$ & Stufe 2 \\
\hline
\end{tabular}

Tab. 1: Einsatzszenarien von Geschäftsanalysen

Initiative zur Geschäftsarchitektur der AXA Winterthur positioniert sich hier im Rahmen der folgenden Gesamtziele:

- Es soll eine erhöhte Transparenz bezogen auf das Geschäftsmodell gewonnen werden. Es gilt, Zusammenhänge, die für die Abwicklung des Geschäfts zentral sind, zu verstehen und systematisch zu kommunizieren.

- Es gilt, zielgerichtet Veränderungen im Geschäftsmodell (i.S. einzelner Anwendungsfälle) zu lokalisieren, um deren Auswirkungen abschätzen zu können. Dies stellt eine wichtige Grundlage für eine koordinierte Weiterentwicklung der fachlichen und IT-Architekturen dar.

- Es gilt, aufzuzeigen, welche Geschäftsaktivitäten oder Geschäftsprozesse in gleicher Art und Weise ausgeführt werden können. Ziel ist es dabei, eine Konsolidierung und Standardisierung (Industrialisierung) der Prozesse unterstützen zu können.

Im Rahmen der Initiative zur Geschäftsarchitektur wurden exemplarisch die folgenden Einsatzbeispiele mit Bezug zur Geschäftsanalyse identifiziert.

\subsection{Geschäftsanalyse aus Sicht der strategischen Unternehmensführung}

Die strategische Unternehmensführung befasst sich mit der langfristigen und grundsätzlichen Ausrichtung des Gesamtversicherungsunternehmens. Sie koordiniert dazu die großen
Unternehmensbereiche entlang der Wertschöpfungskette. Eine Grundlage für die Strategiearbeit der AXA Winterthur bilden strategische Initiativen, die drei übergreifende Stoßrichtungen - Stärkung des bestehenden $\mathrm{Ge}$ schäfts, Erschließung neuer Geschäftsfelder und Berücksichtigung von Mitarbeiterengagement und Kundenfokus - adressieren. Die Herausforderung in der strategischen Unternehmensführung berührt die Geschäftsanalyse u.a. in Form folgender Fragestellungen:

- Welche Geschäftsfunktionen sollen aufgrund veränderter Rahmenbedingungen in einem bestimmten Markt hinsichtlich Kosten, Qualität und Verarbeitungsgeschwindigkeit angepasst werden?

- Ist es möglich, bei gleichbleibender Qualität die Anzahl der Kunden für eine Marktleistung zu erhöhen?

- Kann eine bestimmte Marktleistung auch für andere Kundensegmente oder als Leistungsbündel angeboten werden?

Die Geschäftsarchitektur stellt zu diesem Zweck eine konzeptionelle Sichtweise auf strategische Initiativen dar und fungiert als Analyseinstrument für die strategische Unternehmensführung. Mit ihrer Hilfe ist es u.a. möglich, die Geschäftsstrategie als formulierte Initiativen auf die funktionsorientierten Kernfähigkeiten der AXA Winterthur, dargestellt in Form von Business Capabilities (Geschäftsfähigkeiten), abzubilden. Das Projektportfolio kann bezüglich 
seiner Strategiekonformität beurteilt werden. Abhängigkeiten zwischen Projekten werden erkannt und somit die Risiken einer inkonsistenten Weiterentwicklung einer Geschäftsfähigkeit (z. B. Manage sales partners and alliances) verringert. Die Geschäftsanalyse erlaubt es ebenfalls, Ressourcenengpässe zu identifizieren. Dies ist relevant, wenn Initiativen die Kapazitäten derselben IT-Abteilung binden, z. B. sobald Modifikationen an denselben Applikationen geplant sind. Im Ergebnis erlaubt die Geschäftsanalyse, das Projektportfolio entsprechend der Geschäftsstrategie zu beurteilen und im selben Zug das Applikationsportfolio aktiv zu bewirtschaften. Dieses Beispiel legt den Fokus auf die Nachhaltigkeit als Ziel der Geschäftsanalyse. Es wird die langfristige Ausrichtung des Unternehmens in den Mittelpunkt der Analyse gestellt, auch mit dem Wunsch, zukünftige Geschäftspotenziale identifizieren zu können.

\subsection{Geschäftsanalyse aus Sicht der Produktentwicklung}

Das Versicherungsprodukt im Sinne einer Marktleistung wird durch Informationen abgebildet. Aus diesem Grund weist die Versicherungsschutzproduktion eine starke informationstechnische Komponente auf. Die Produktentwicklung ist ein zentrales Thema in der Versicherungswirtschaft. Ausgehend von früheren Segmentierungsstrategien wie der Trennung nach Sparten wurden zahlreiche Legacy-Systeme für die Produktdefinition und -verwaltung aufgebaut. Eine aktuelle Herausforderung besteht darin, auf IT-Ebene den Austausch von Informationen über verschiedene Unternehmensbereiche und Organisationseinheiten hinweg zu ermöglichen. Dazu können u.a. folgende für die Geschäftsarchitektur relevanten Gestaltungsprinzipien formuliert werden:

- Die IT-Beteiligung soll sich auf ein Minimum beschränken und die Fachseite in die Lage versetzen, Produkte zu entwickeln und zu verwalten.
- Die Produktmerkmale sollen separiert von Vertragsverwaltungssystemen definiert und vorgehalten werden.

- Die Aktivitäten im Produktmanagement sollen verstärkt zentral abgewickelt werden, um den Fokus auf den Kundenwunsch zu setzen.

Die Geschäftsanalyse verknüpft die verschiedenen Sichten rund um das Gestaltungsobjekt Produkt wie z. B. Kundenproduktsicht, vertriebsorientierte, tarifliche und Produktentwicklersicht. Es wird deutlich, welche Elemente Einfluss auf das Versicherungsprodukt haben, $d$.h., welche Attribute ein bestimmtes Produkt besitzt und welche Rollen im Produktentwicklungsprozess beteiligt sind. Zusätzlich wird sichtbar, welche Prozesse und Applikationen im Rahmen der Produktentwicklung involviert sind und welche Abhängigkeiten zwischen ihnen bestehen. Bezogen auf die Dimensionen der Gestaltung von Versicherungsprodukten sind folgende Analysen von Bedeutung:

- Wie ist das Produkt bezogen auf das Produktportfolio positioniert?

- Welches Markt- bzw. Kundensegment kann angesprochen werden, welcher Absatzkanal wird angeboten?

- Welche ggf. versicherungsfremden Zusatzleistungen sind Bestandteil des Produktes und wie ist die Schnittstelle zu einem Geschäftspartnerprozess?

Steht ein flexibles Produktmodell zur Verfügung, so erlaubt die Geschäftsanalyse das Lebenszyklusmodell von Produkten dem Modell der Geschäftsfähigkeiten der AXA Winterthur zuzuordnen, um funktionale Anforderungen aus Produktsicht mit den zentralen, definierten Fähigkeiten der AXA Winterthur abzugleichen. Die Geschäftsanalyse soll gewährleisten, dass die erforderlichen Informationen in der Produktentwicklung bekannt und bezogen auf den Produktlebenszyklus zum richtigen Zeitpunkt verfügbar sind. Die Analysen in diesem Beispiel unterstützten die Wandlungsfähigkeit als Ziel 
der Geschäftsanalyse, z. B. in Form flexibler Produktmodelle für die Produktentwicklung.

\subsection{Werkzeugunterstützte Geschäfts- analyse}

In den fachlichen Abläufen der AXA Winterthur wird eine Vielzahl von Informationen produziert oder bearbeitet. Verteilt über das Unternehmen pflegen verschiedene Organisationseinheiten und Projekte individuelle Vorlagen und Arbeitsergebnisse z. B. in Form von ExcelListen und Dokumentenablagen. Jeder Vorlage und jedem Ergebnis liegt ein Verständnis zugrunde, wie derartige Vorlagen oder Arbeitsergebnisse aufgebaut sind. Bestenfalls sind solche Ad-hoc-Verständnisse in Form von Glossaren, Richtlinien oder Templates dokumentiert. Oftmals sind sie jedoch nur implizit vorhanden. Daraus folgt, dass eine Vielfalt ähnlicher Ergebnisse mit Bezug zur Geschäftsarchitektur und unterschiedlicher Ausprägung erzeugt und gepflegt wird. Aus diesem Grund wiederholen sich Diskussionen zu denselben fachlichen Themen, und Zusammenhänge zwischen voneinander abhängigen oder ähnlichen Ergebnissen werden nicht erkannt.

Die Geschäftsanalyse unterstützt hierbei, indem Ergebnisse als Output von Prozessen betrachten werden. So wird ersichtlich, welche Ergebnisse in welchen Aktivitäten produziert und weiterverwendet werden. Außerdem ist erkennbar, welche fachlichen Sachverhalte in welchen Ergebnissen modelliert sind. Diese Überlegungen sind Voraussetzung für die Erstellung von gemeinsam verwendeten Templates und Dokumentstrukturen. Um die ArchitekturAssets, z. B. strategische Initiativen, Geschäftsfähigkeiten und Informationssysteme (IS), zu bewirtschaften, ist die Geschäftsanalyse eng an die Kernprozesse des Architekturmanagements gekoppelt. Das Lifecycle-Management der Architektur-Assets wird werkzeugunterstützt betrieben, da nur so Zusammenhänge und $A b$ hängigkeiten automatisiert aufgedeckt und bearbeitet werden können. Das Modell der Ge- schäftsarchitektur als Basis der Geschäftsanalyse visualisiert diese Überlegungen. Ein werkzeugunterstütztes Management der Geschäftsarchitektur zielt auf die Speicherung der Metainformationen über Architektur-Assets in einem Repository ab. So können im Rahmen der Geschäftsanalyse fachlich orientierte Kennzahlen automatisiert generiert werden. Die werkzeugunterstützte Geschäftsanalyse hilft außerdem dabei, die Ausdehnung von Pflegeund Nutzungsprozessen auf andere Architekturbereiche bewusst zu steuern. Eine solche Ausdehnung wurde exemplarisch durchgeführt, indem in Ergänzung zu den bereits gepflegten IT- und IS-Daten neu auch Daten der Geschäftsarchitektur erfasst und mit den Applikationsdaten verknüpft wurden. Die Geschäftsanalyse fokussiert hierbei auf die Konsistenzerhaltung, u. a. indem Pflege- und Nutzungsprozesse für Architekturbereiche vereinheitlicht werden.

\section{Gesamtmodell der Geschäftsanalyse}

Anhand der Ziele der Geschäftsanalyse auf Basis der Unternehmensarchitektur wird im Folgenden aufgezeigt, welche Analysetypen sich generell differenzieren lassen. Dazu wird ein Gesamtmodell zur Geschäftsanalyse vorgeschlagen, in dem die Gestaltungsobjekte der Geschäftsarchitektur den identifizierten Analysetypen mithilfe exemplarischer Analysen gegenübergestellt werden.

\subsection{Typen der Geschäftsanalyse}

Geschäftsanalysen im Kontext der Unternehmensarchitektur lassen sich in technische und fachliche Analysetypen unterscheiden. Technische Analysen fokussieren auf die topologischen Eigenschaften und Abhängigkeiten der Gestaltungsobjekte. Es gilt z. B., Lücken und Redundanzen zwischen Gestaltungsobjekten zu identifizieren (Abdeckungsanalyse), um etwa den Automatisierungsgrad von Geschäftsprozessen zu bestimmen. Ebenso verdeutlichen 
sie Gemeinsamkeiten und Unterschiede der Gestaltungsobjekte (Heterogenitätsanalyse) zum Zweck der Homogenisierung und Standardisierung. Als klassisch fachliche Analysen werden u.a. Compliance- und Wirtschaftlichkeitsanalysen zusammengefasst. Sie weisen z. B. den Umsetzungsgrad gesetzlicher Auflagen nach und unterstützen bei der Zuordnung von Entwicklungsressourcen zu Projekten. Die folgende Abbildung 3 zeigt die hier nicht abschließend aufgezählten Typen der Geschäftsanalyse im Kontext der Unternehmensarchitektur.

\subsection{Breite und Tiefe der Geschäftsanalyse}

Neben der Zuordnung zu Zielen im Rahmen der Geschäftsanalyse können die Analysetypen hinsichtlich adressierter Gestaltungsobjekte der Unternehmensarchitektur betrachtet werden.
Dazu wird das eingangs vorgestellte Verständnis von Unternehmensarchitekturen als Ebenenmodell den identifizierten Analysetypen gegenübergestellt. Abbildung 4 illustriert diese Zuordnung, indem für ausgewählte Analysetypen konkrete Beispielanalysen auf den Ebenen der Geschäftsarchitektur aufgeführt werden.

Es wird deutlich, dass die Geschäftsanalyse auf allen relevanten Ebenen der Unternehmensarchitektur sinnvoll verankert werden kann. Die Pfeildarstellung bezieht sich dabei auf die jeweiligen Bezugspunkte einer Analyse. Eine Unterteilung in Gesamt- und Partialanalysen spiegelt dabei die Möglichkeit wider, Analysen über mehrere Ebenen und Teilarchitekturen hinweg zu positionieren.

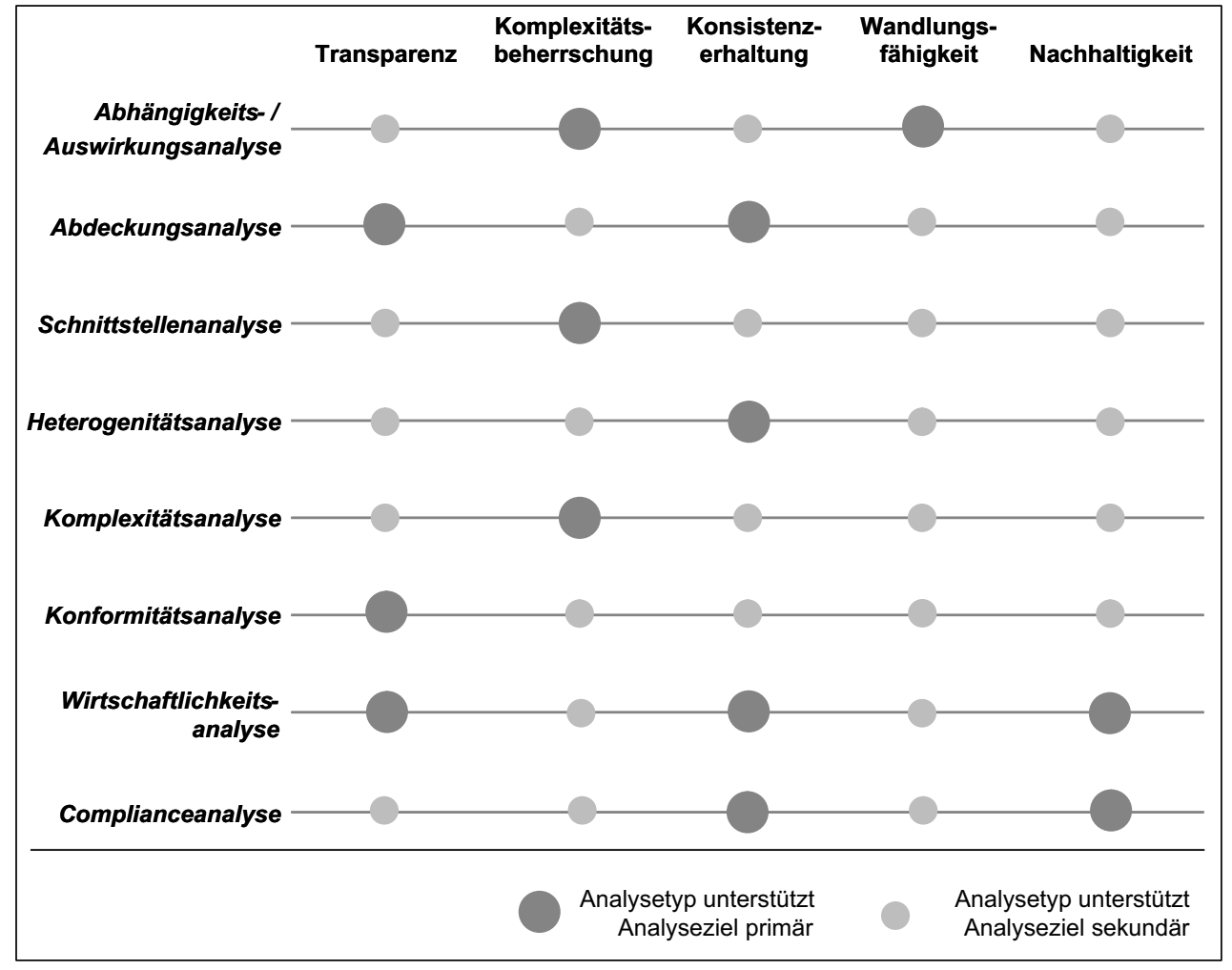

Abb. 3: Analysetypen und Analyseziele 


\begin{tabular}{|c|c|c|c|c|c|}
\hline \multicolumn{2}{|c|}{$\begin{array}{l}\text { Analysety } \\
\text { Gestaltungsobjekte }\end{array}$} & \multirow[t]{4}{*}{$\begin{array}{l}\text { Abhängigkeits- und } \\
\text { Auswirkungsanalyse }\end{array}$} & \multirow{4}{*}{\begin{tabular}{|c|} 
Abdeckungsanalyse \\
1 \\
\end{tabular}} & \multirow{4}{*}{\begin{tabular}{|c|} 
Konformitätsanalyse \\
\\
7 \\
\end{tabular}} & \multirow{3}{*}{$\begin{array}{c}\begin{array}{c}\text { Wirtschaftlichkeits- } \\
\text { analyse }\end{array} \\
6\end{array}$} \\
\hline 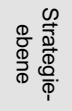 & $\begin{array}{l}\text { Produkte, } \\
\text { Marktsegmente, } \\
\text { Geschäftspartner, } \\
\text { Strat. Projekte }\end{array}$ & & & & \\
\hline 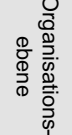 & $\begin{array}{l}\text { Geschäftsprozesse, } \\
\text { Geschäftsfkt., } \\
\text { Rollen, Org.- } \\
\text { Einheiten }\end{array}$ & & & & \\
\hline 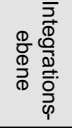 & $\begin{array}{l}\text { Domänen, } \\
\text { Applikationen, } \\
\text { fachliche Services }\end{array}$ & & & & \\
\hline 1 & \multicolumn{5}{|c|}{ Welche Produkte bedienen welches Marktsegment? } \\
\hline 2 & \multicolumn{5}{|c|}{ Welche Organisationseinheit trägt in welchem Umfang zur Erreichung von Unternehmenszielen bei? } \\
\hline 3 & \multicolumn{5}{|c|}{ Welche Marktleistungen sind bei Nichtverfügbarkeit einer bestimmten Applikation betroffen? } \\
\hline 4 & \multicolumn{5}{|c|}{ Welche strategischen Vorhaben benötigen die gleichen IS-Ressourcen bzw. modifizieren die gleichen Applikationen? } \\
\hline 5 & \multicolumn{5}{|c|}{ Ist die Rollenstruktur des Prozesses korrekt in der Berechtigungsstruktur der Applikation abgebildet? } \\
\hline 6 & \multicolumn{5}{|c|}{ Welche Umsatz-/Deckungsbeitragsvolumina hängen an welchem Prozess bzw. an welcher Applikation? } \\
\hline 7 & \multicolumn{5}{|c|}{ Wo kann Prozessstandardisierung stattfinden, d.h., welche Teilprozesse sind prozessübergreifend gleich gestaltet? } \\
\hline
\end{tabular}

Abb. 4: Beispiele für Geschäftsanalysen und Ebenen der Geschäftsarchitektur

\subsection{Umsetzung der Geschäftsanalyse}

Die Umsetzung der Geschäftsanalyse orientiert sich am möglichen Nutzenpotenzial und den verursachten Kosten der einzelnen Analysen. Somit muss bei der Implementierung neben der rein fachlichen und technischen Unterscheidung der Analysetypen auch das Potenzial von Gesamt-, respektive Partialanalysen differenziert werden. Hierbei kann bei Geschäftsanalysen auf Basis der Unternehmensarchitektur zwischen einfachen Reports und komplexen Auswertungen mit mehrstufigen Abhängigkeiten unterschieden werden. Diese Differenzierung ist notwendig, um beim Aufbau eines Repositories für Unternehmensarchitekturen Auswertungen anspruchsgruppenorientiert aufzubereiten. Oftmals werden im operativen Geschäft nur die grundlegenden Informationsbedarfe in Form einfacher Auflistungen z. B. der Gestaltungsobjekte nachgefragt. Komplexe Auswertungen mit mehrstufigen Abhängigkeiten über verschiedene Gestaltungsebenen werden i. d. R. weniger häufig genutzt. Der Grund liegt im eher strategischen Charakter ihrer Ergebnisse, die im Allgemeinen nicht Gegenstand häufiger Diskussionen und Änderungen im Unternehmen sind. Somit wird oft einfachen Reports der Vorzug gegenüber komplexen und damit erklärungsbedürftigen Auswertungen gegeben. Dies gilt insbesondere dann, wenn sich die Implementierung bzw. Werkzeugunterstützung der Geschäftsanalyse noch im Aufbau befindet.

\section{Lerneffekte und weitere Entwicklung}

Zusammenfassend kann festgestellt werden, dass sich die Umsetzung der Unternehmensarchitektur nicht auf die passive (Nach-)Führung eines unternehmensweiten Repositories für wichtige Gestaltungsobjekte beschränken sollte, sondern dass auf Grundlage einer Unternehmensarchitektur mit "Business-to-IT«-Fokus eine Vielzahl sinnvoller Geschäftsanalysen möglich wird. Dabei darf aus wirtschaftlichen Gründen der Gesamtaufwand zur Durchführung von aussagekräftigen Geschäftsanalysen 
den generierten Nutzen nicht übersteigen. Gegenwärtig wird die Geschäftsarchitektur als Ausgangsbasis für die Geschäftsanalyse in der Praxis jedoch nicht in dem Maße berücksichtigt, wie es die angeführten Nutzenpotenziale nahelegen. Die Geschäftsanalyse liefert Auswertungen und Ergebnisse für die Entscheidungsunterstützung in zahlreichen Aufgabengebieten [Deelmann 2007; Kilov 2002]:

- Das Verständnis für fachliche Arbeitsergebnisse kann vereinheitlicht werden, indem die Geschäftsarchitektur als konsistente Grundlage für Analyse-, Dokumentations- und Schulungszwecke genutzt wird.

- Durch die Geschäftsanalyse werden Ansatzpunkte für Prozessveränderungen sowie die Konsolidierung und Standardisierung von Geschäftsprozessen sichtbar.

- Die Geschäftsanalyse unterstützt die Organisation dabei, Wechselwirkungen zwischen dem Geschäftsmodell und seiner Umsetzung durch Informationstechnologie zu erkennen und zu steuern.

Insgesamt bleibt kritisch festzuhalten, dass gerade die Pflege und Aktualität der benötigten Daten der Geschäftsarchitektur für Geschäftsanalysen von hoher Bedeutung sind. Weiterer Forschungs- und Untersuchungsbedarf ergibt sich hinsichtlich der Einbettung der Geschäftsanalyse in die bestehenden Strukturen und Abläufe der klassischen Unternehmenssteuerung. Der Wirkungsgrad der Geschäftsanalyse im Kontext der Unternehmensarchitektur ist in besonderem Maße abhängig von der positiven Wahrnehmung der Unternehmensarchitektur auch über die Grenzen des IT-Bereichs einer Organisation hinaus.

\section{Literatur}

[Baumöl 2006] Baumöl, U.: Methodenkonstruktion für das Business-IT-Alignment. In: Wirtschaftsinformatik, 48. Jg., 2006, Nr. 5, S. 314-322.

[Deelmann 2007] Deelmann, T.: Geschäftsmodellierung - Grundlagen, Konzeption und Integration. Logos, Berlin, 2007.

[Keller 2007] Keller, W.: IT-Unternehmensarchitektur: Von der Geschäftsstrategie zur optimalen IT-Unterstützung. dpunkt.verlag, Heidelberg, 2007.

[Kilov 2002] Kilov, H.: Business Models - A Guide for Business and IT. Prentice Hall, Upper Saddle River, 2002.

[Lankhorst 2005] Lankhorst, M.: Enterprise Architecture at Work: Modelling, Communication and Analysis. Springer-Verlag, Berlin u.a., 2005.

[Niemann 2005] Niemann, K. D.: Von der Unternehmensarchitektur zur IT-Governance: Leitfaden für effizientes und effektives IT-Management. Vieweg, Wiesbaden, 2005.

[The Open Group 2007] The Open Group: The Open Group Architecture Framework TOGAF - 2007 Edition (Incorporating 8.1.1). Zaltbommel, 2007.

[Winter \& Fischer 2007] Winter, R.; Fischer, R.: Essential Layers, Artifacts, and Dependencies of Enterprise Architecture. In: Journal of Enterprise Architecture, 3. Jg., 2007, Nr. 2, S. 7-18.

[Winter et al. 2007] Winter, R.; Bucher, T.; Fischer, R.; Kurpjuweit, S.: Analysis and Application Scenarios of Enterprise Architecture - An Exploratory Study. In: Journal of Enterprise Architecture, 3. Jg., 2007, Nr. 3, S. 33-43.

Dipl.-Wirtsch.-Inf. Christian Riege

Prof. Dr. Robert Winter

Universität St. Gallen

Institut für Wirtschaftsinformatik

Müller-Friedberg-Str. 8

$\mathrm{CH}-9000$ St. Gallen

\{christian.riege, robert.winter\}@unisg.ch

www.iwi.unisg.ch

lic. oec. HSG Matthias Stutz

Stevens Institute of Technology

Hoboken, NJ 07030, USA

matthias.stutz@stevens.edu

www.stevens.edu 